\title{
ESTRATEGIAS DE MARKETING EN UNA BIBLIOTECA PÚBLICA DE SUBURBIO: BIBLIOTECA FONT DE LA MINA
}

\author{
Montserrat Espuga-Condal
}

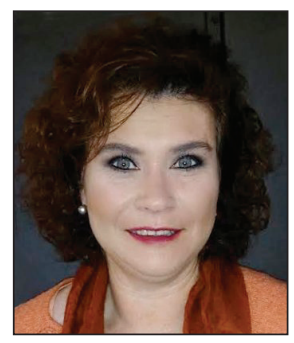

Montserrat Espuga-Condal es graduada en información y documentación y diplomada en biblioteconomía y documentación por la Universitat de Barcelona, postgrado en sistemas de información en la empresa por el Institut Català de Noves Professions y mediador intercultural por el Ministerio de Sanidad, Servicios Sociales e Igualdad y Unión Romaní. Ha trabajado en las empresas SiemensCerberus y CIFA en gestión de la documentación técnica, y en bibliotecas públicas, dirigiendo desde 2011 la Biblioteca Font de La Mina de Sant Adrià de Besòs (Barcelona). Ha sido miembro de la sección de Public Libraries de la IFLA (2013-2017).

http://orcid.org/0000-0001-6907-6212

Biblioteca Font de la Mina C/ Ponent, 25. 08930 St. Adrià de Besòs (Barcelona), España espugacm@diba.cat

\section{Resumen}

Se presentan las técnicas de negociación colectiva, análisis de situación y plan de marketing aplicados en una biblioteca pública de un barrio considerado durante años como marginal. La biblioteca forma parte del plan de transformación del barrio. Con la aplicación de las estrategias empresariales que se exponen, se ha conseguido convertirla en una influyente instalación de referencia del barrio.

\section{Palabras clave}

Marketing mix; Estrategias de marketing; Promoción; Bibliotecas; Bibliotecas públicas; DAFO; Negociación colaborativa; Etnias; Gitanos; Barrio periférico; Zona suburbana; Biblioteca Font de La Mina, Barcelona.

\section{Title: Marketing strategies in a suburban public library. Biblioteca Font de la Mina}

\begin{abstract}
Collective bargaining techniques, situation analysis and a marketing plan implemented in a public library are discussed. The library, located in a neighborhood that has long been considered marginal, is part of the transformation plan for the district. With the implementation of the business strategies set out in this article, we have managed to turn the library into an influential resource for the neighborhood.
\end{abstract}

\section{Keywords}

Marketing mix; Marketing strategies; Libraries; Public libraries; Negotiation; SWAT; Gypsies; Suburb; Suburban area; Font de La Mina Public Library, Barcelona.

Espuga-Condal, Montserrat (2015). “Estrategias de marketing en una biblioteca pública de suburbio: Biblioteca Font de la Mina". El profesional de la información, v. 24, n. 1, enero-febrero, pp. 39-43.

http://dx.doi.org/10.3145/epi.2015.ene.05

\section{Introducción}

Las bibliotecas, como otras entidades que ofrecen servicios al público, tienen que realizar acciones de marketing para atraer la atención de los usuarios, que hoy en día se ven bombardeados por múltiples opciones de formación, cultura y ocio.

A menudo se aplican técnicas y estrategias comerciales sin ser conscientes de ello, como por ejemplo ofrecer a los usuarios las novedades de forma diferente: en un expositor, en una mesa en la entrada de la biblioteca, etc. Es la misma estrategia que utilizan las librerías al destacar en mesas fácilmente accesibles y con varios ejemplares los últimos libros publicados.

En general, para llevar a cabo esas acciones se cuenta con un pequeño presupuesto pero en los años recientes muchas bibliotecas carecen de tal asignación debido a los recortes presupuestarios. Por ello, en 2012 en la biblioteca pública Font de La Mina se planteó la posibilidad de no programar más acciones de fomento de la lectura como solía hacerse para ocasiones extraordinarias (Navidad, Halloween, etc.) pues sin presupuesto no se podía contratar ningún profe- 
sional. Sin embargo todo el equipo de la biblioteca decidió reinventarse y aplicar el conocido slogan del presidente norteamericano Barack Obama en su campaña electoral de 2008: Yes, we can!. El primer paso fue establecer alianzas colaborativas que han permitido organizar la mayoría de los actos: por ejemplo, la decoración de Navidad se hizo con materiales procedentes de las calles engalanadas para las fiestas de agosto del Barrio de Gràcia de Barcelona; y en verano a los niños se les ofrecieron puzzles y juegos educativos procedentes de una fundación de reinserción del barrio. Otra acción de marketing fue el sorteo de una cesta de lectura con jamón incluido (El periódico, 2012).

\section{No existen métodos replicables en todos los entornos, sino un denominador co- mún que es la necesidad de flexibilidad y adaptación, y de reinventarse continua- mente}

\section{Descripción del entorno}

La biblioteca pública Font de La Mina se encuentra en el barrio de La Mina del municipio de Sant Adrià del Besòs, que limita con los de Santa Coloma de Gramenet, Badalona y Barcelona. El barrio está formado por veinte bloques de edificios que conforman un total de 2.721 viviendas y 181 locales. En los últimos años las áreas colindantes, especialmente El Fòrum y la parte final de la Av. Diagonal, se han desarrollado urbanísticamente y han generado una nueva actividad hotelera, lúdica, deportiva y comercial, lo que ha situado La Mina en el mapa del Área Metropolitana de Barcelona. Durante décadas fue una zona aislada físicamente, rodeada de zonas industriales o sin uso definido.

El barrio se originó como resultado de una actuación gubernamental iniciada en 1969 con el objetivo de erradicar varios núcleos de chabolas. En 1972 se inició la construcción de las 1.871 viviendas de La Mina Nueva alcanzando los 15.000 habitantes, y en 1.975 se construyeron otras 2.029 viviendas. A finales de los años 90 el Ayuntamiento de Sant Adrià,

la Generalitat de Catalunya, la Diputación de Barcelona y el Ayuntamiento de Barcelona aprobaron el Plan de Transformación del barrio de La Mina para el período 2000-2010 con una prórroga posterior de cinco años (2011-2015), creando el Consorci del Barri de La Mina para llevarlo a cabo.

El padrón municipal a 1 de enero de 2014 cifra la población residente en 9.777 personas. En cuanto al nivel de formación, en la población entre 18 y 64 años:

- un $43 \%$ tiene el graduado escolar;

- un $25 \%$ no ha acabado los estudios básicos;

- el 22,5\% ha superado el bachillerato superior; y

- solamente un $0,5 \%$ ha realizado estudios universitarios.

\section{La Biblioteca Font de La Mina}

Comparte espacio con el Espai Cultural Font de La Mina y su característica principal es la labor social que cumple. Se trata de una biblioteca de proximidad dentro de la Red Urbana de Sant Adrià de Besòs para un área de influencia de 15.000 habitantes.

Algunos datos del centro:

- superficie: $2.122 \mathrm{~m}^{2}$;

- 22 ordenadores de uso público;

- 32.000 volúmenes;

- 56.714 visitas en 2013;

- 3.530 usuarios en enero de 2014.

El 70\% de los usuarios son de etnia gitana. La biblioteca tiene un perfil de usuario fiel y que aprecia la atención personalizada que se le ofrece. Cuando se inauguró en 2009, la mayoría de los habitantes del barrio desconocía cuáles eran el funcionamiento y los servicios de una biblioteca pública, puesto que no existía ninguna referencia de instalación similar (Placer, 2010).

El valor social es el más destacable del conjunto de servicios que ofrece la biblioteca, y es un valor intangible, difícil de identificar y de cuantificar. Podemos resaltar la adaptación de las normas a un tipo de usuarios no habituados a este tipo de servicio. Por ejemplo, se permite saldar las deudas de pérdidas o desperfecto de libros y muy especialmente de DVDs con otros títulos similares.

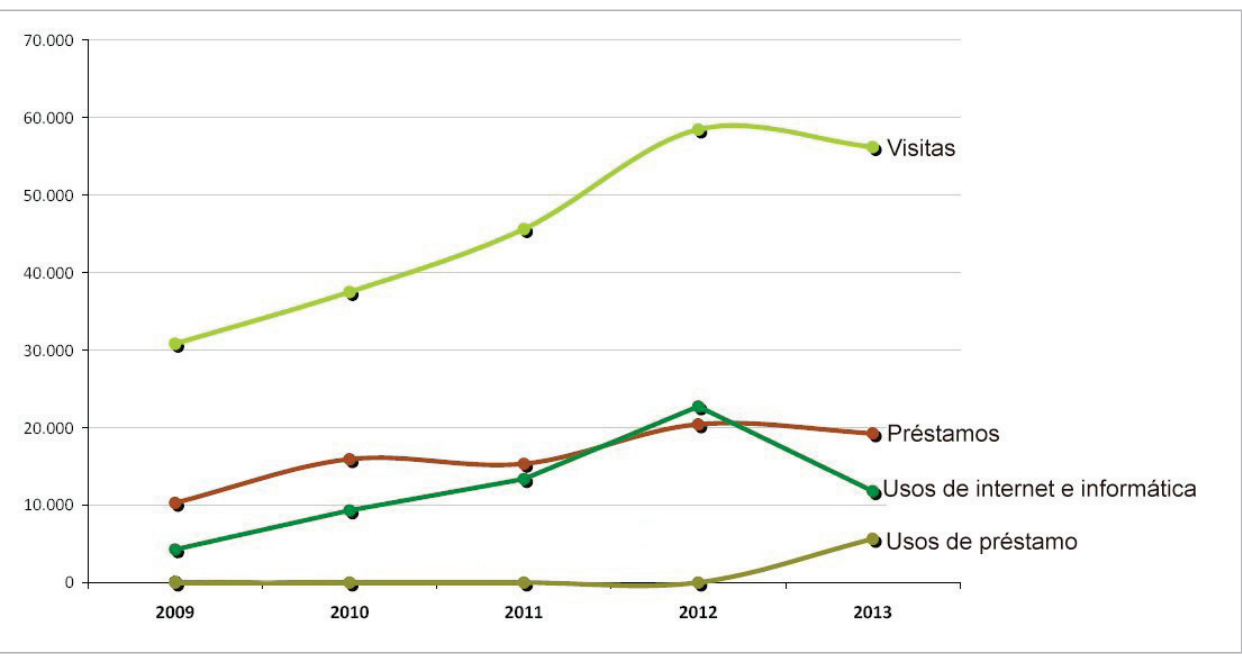

Figura 1. Evolución de los servicios 2009-2013. Fuente: Gerència del Servei de Biblioteques. Diputació de Barcelona

\section{Estrategias y medios utilizados}

\subsection{Trabajo en equipo}

El equipo de la biblioteca está formado por una directora, una bibliotecaria, cuatro técnicos auxiliares, una dinamizadora y un conserje. Trabajan por objetivos y proyectos comunes, no por tareas asignadas. Todo el equipo colabora en todas las acciones, teniendo cada proceso dos personas de referencia y una de reserva, 
de manera que no se paralice nada por falta de personal. Los medios que se utilizan son:

- Distribución y asignación de los procesos: zona infantil, zona de adultos, zona de estudio, zona de fomento de la lectura y la comprensión lectora (Caçadors de llibres).

- Calendario común de Microsoft Outlook donde cada miembro del equipo anota su agenda y se incluye toda la programación anual de la biblioteca.

- Ordenadores compartidos: todos los ordenadores están accesibles a todos los miembros, aunque cada miembro trabaja habitualmente en uno concreto.

- Información compartida: en el ordenador central de la red interna (Xemeneia) se introducen y comparten los datos y los materiales de formación de todos los miembros del equipo, fotos de bibliotecas visitadas, etc.

\subsection{Negociación}

La técnica que se aplica es la negociación colaborativa, también conocida como "ganar-ganar", basada en que ambas partes deben obtener beneficios (Fuller, 1998).

Aunque podría considerarse mediación, el ejemplo que se expone se ha tratado desde el punto de vista de la negociación colaborativa. Las negociaciones no siempre son con una persona, pueden ser con una familia. Un ejemplo son los trabajos a la comunidad que se firman en la biblioteca con el Departament de Justícia de la Generalitat de Catalunya. Se trata de horas de trabajo comunitario que se realizan en la biblioteca. No sería destacable si no tuviese particularidades, porque son muchas las bibliotecas que acogen personas que realizan sus prestaciones a la comunidad. En nuestro caso consideramos que debemos ser consecuentes con nuestro entorno, y adaptar los trabajos a realizar al perfil de la persona. Por ejemplo, en una ocasión, una familia con 5 hijos, uno de ellos un bebe de 2 meses, debían realizar cada uno de los padres 30 horas de trabajo a la comunidad por hurto en una superficie comercial. Estas personas realizaron los trabajos en la sala infantil de la biblioteca junto a su bebé, y procuramos compaginar la dinámica de un recién nacido con las tareas de la biblioteca, básicamente recortar materiales para los talleres. El resultado de esta colaboración ha sido, por parte de la biblioteca, la captación de nuevos usuarios y la proyección de una imagen positiva e integradora, y por parte de la familia, la dignificación de su trabajo (elemento esencial si se trata de gitanos).

\subsection{Estrategia empresarial}

\subsubsection{Determinar dónde estamos}

Se trata de analizar la situación tanto interna (fortalezas y debilidades) como externa (oportunidades y amenazas), es decir, elaborar un DAFO (Learned, 1969), que se muestra a continuación.

\section{Oportunidades}

- instalaciones de la futura Ciudad de la Economía del Conocimiento en terrenos municipales de Sant Adrià de Besòs, Key Barcelona (La vanguardia, 2012);

- la población del área de influencia está evolucionando hacia la multiculturalidad;

- instalaciones de la Escuela de Ingeniería Técnica de la UPC
(Angulo; Benvenuty, 2012), que finalizan en 2015; - la biblioteca tiene un alto componente social.

\section{Amenazas}

Durante años el barrio de La Mina se ha considerado marginal y ha sido estigmatizado. Actualmente está al final de su transformación, pero todavía se asimila el barrio con delincuencia, tráfico de drogas o violencia. Paralelamente, en la parte posterior de la biblioteca están situadas temporalmente las instalaciones de ayuda y control de drogodependientes, y en la proximidad se encuentra el edificio de la Calle Venus que tiene una problemática no solucionada en el Plan de Transformación del Barrio de La Mina (Moya, 2013).

\section{Fortalezas}

- buen nivel de comunicación y colaboración con los agentes y las entidades del barrio;

- es el espacio cultural de referencia en el barrio;

- donaciones de libros y otros materiales que mejoran la colección y fomentan el trabajo en red dentro de la Xarxa de Biblioteques Municipals;

- inicio del programa Lecxit de la Fundación Jaume Bofill, de colaboración de la biblioteca pública y las escuelas para el fomento de la lectura y la comprensión lectora.

\section{Debilidades}

La mayor es la falta de presupuesto municipal para las actividades de difusión de la lectura y para la adquisición de fondos documentales, y en menor medida la falta de formación del personal en aspectos relacionados con la mediación y el trabajo social.

\subsubsection{Determinar dónde queremos llegar}

Nuestra misión es responder al motivo de existencia de la biblioteca, que es en un $70 \%$ labor social y en un $30 \%$ cultural.

Los valores que ofrecemos son acceso público y gratuito a las instalaciones y todas las fuentes de información disponibles adaptadas al entorno, que en nuestro caso se materializan, por ejemplo, en no desconectar el router para que los usuarios puedan acceder a internet fuera del horario de biblioteca, o el respeto a las culturas, en especial la gitana.

El instrumento utilizado para alcanzar nuestros objetivos es el Plan estratégico, basado en una serie de decisiones que se deben tomar para responder a las preguntas siguientes:

- ¿Qué productos y servicios queremos ofrecer?: Todos los de la biblioteca pública, más una atención personalizada.

- ¿Qué demandas del mercado queremos/podemos satisfacer?: Deseamos poder acercar la información a nuestros usuarios, con el fin de evitar al máximo la brecha digital.

- ¿A qué segmento de clientes deseamos atender?: Nos centramos en los habitantes del barrio.

- ¿Qué tecnología vamos a utilizar?: Nos centramos en las técnicas de venta.

- ¿Qué forma de distribución aplicaremos?: Contestamos la pregunta con la adaptación de cada uno de los servicios a nuestros usuarios.

- ¿En qué área geográfica?: Barrio de La Mina y área colindante. 


\section{Marketing}

El marketing engloba los conceptos de comunicación, promoción y publicidad (Santesmases, 2012). El instrumento para materializarlo es el Plan de marketing, para el cual en primer lugar se debe identificar el mercado y segmentar los destinatarios del servicio que se quiere promocionar:

- Población de referencia: datos socio-demográficos, edad, género, diversidad étnica, nivel de estudios.

- Usuarios/no usuarios; grupos de edad y sexo, uso de los servicios.
- Organismos de la comunidad: educativos, sanitarios, voluntariado.

- Destinatarios: niños, jóvenes, adultos, mayores, madres, mujeres.

- Comercios y empresas de la zona, y su área de influencia.

A continuación, y especialmente mediante la observación, debe identificarse el comportamiento del consumidor con la utilización de técnicas de psicología de ventas para crear la necesidad.

Hemos detectado las necesidades siguientes:

Tabla 1. Indicadores cualitativos resultado del marketing mix

\begin{tabular}{|c|c|}
\hline 4P - Marco global & 4P en la Biblioteca Font de La Mina \\
\hline \multicolumn{2}{|r|}{ Producto } \\
\hline $\begin{array}{l}\text { Calidad } \\
\text { Características } \\
\text { Diseño } \\
\text { Nombre de la marca } \\
\text { Tamaños } \\
\text { Servicios } \\
\text { Garantías } \\
\text { Devoluciones }\end{array}$ & $\begin{array}{l}\text {-Calidad: fondos bibliográficos seleccionados adaptados al entorno. } \\
\text {-Características: documentos en diferentes formatos, acceso a internet y a ordenadores, etc. } \\
\text {-Diseño: el propio de la biblioteca o red de bibliotecas a la que pertenece. } \\
\text {-Nombre de la marca: el concepto “biblioteca”. } \\
\text {-Tamaños: formas de acceso a los servicios: presenciales, en línea, etc. } \\
\text {-Servicios: todos los que ofrecen las bibliotecas públicas. } \\
\text {-Garantías: prestación de servicios de calidad. } \\
\text {-Devoluciones: retorno de los materiales con el compromiso de mantener su buen estado. }\end{array}$ \\
\hline \multicolumn{2}{|r|}{ Lugar } \\
\hline $\begin{array}{l}\text { Distribución } \\
\text { Canales } \\
\text { Cobertura } \\
\text { Variedad } \\
\text { Localización } \\
\text { Inventario } \\
\text { Transporte }\end{array}$ & $\begin{array}{l}\text { La biblioteca está dividida en } 4 \text { zonas distribuidas en } 2 \text { plantas: } \\
\text {-Planta baja: zona de ocio que contiene la sección de novelas, revistas, música, cómic, viajes, biografías y literatura y zona } \\
\text { infantil. } \\
\text {-Primera planta: zona de estudio y silencio. Los ordenadores están reservados para el estudio. } 4 \text { salas independientes: estu- } \\
\text { dio, tertulia, trabajo en equipo y sala de comprensión lectora Caçadors de Llibres. }\end{array}$ \\
\hline \multicolumn{2}{|r|}{ Promoción y difusión } \\
\hline $\begin{array}{l}\text { Acercamiento psico- } \\
\text { social }\end{array}$ & $\begin{array}{l}\text { La biblioteca tiene en cuenta el alto porcentaje de población de etnia gitana, y adapta algunas de las actividades en conse- } \\
\text { cuencia. } \\
\text { Muchos son miembros de la Iglesia Evangélica Filadelfia. El culto diario se inicia a las } 19 \mathrm{~h} \text {, por lo que la biblioteca procura } \\
\text { programar las actividades infantiles de forma que finalicen sobre esa hora y así los usuarios de la zona infantil pueden } \\
\text { acudir a las dos actividades. } \\
\text { Otro ejemplo es el respeto al duelo de las familias. Los niños menores de } 6 \text { años deben ir a la biblioteca acompañados por } \\
\text { un adulto, pero si los padres no pueden entrar debido al proceso de duelo, se deja entrar al niño siempre que los padres } \\
\text { permanezcan en las inmediaciones de la biblioteca. }\end{array}$ \\
\hline Promoción de ventas & $\begin{array}{l}\text { Todos los servicios de la biblioteca se fomentan por igual, pero con peculiaridades. Actualmente la mayoría de las gestiones } \\
\text { administrativas se realizan por internet. Algunos de los usuarios, especialmente mujeres gitanas, no saben cómo realizar los } \\
\text { trámites, y desde la biblioteca se les asiste. Este tipo de atención personalizada es muy valorada en el barrio; al tratar temas } \\
\text { privados, la discreción del personal de la biblioteca es fundamental. }\end{array}$ \\
\hline Publicidad & $\begin{array}{l}\text { La biblioteca tiene un programa semanal en Radio La Mina donde se cuentan las novedades, las actividades y se lee un } \\
\text { cuento, poesía o fragmento de un libro. El podcast del programa se puede descargar desde el Facebook de la biblioteca o } \\
\text { desde la web de Radio La Mina, http://radiolamina.blogspot.com.es } \\
\text { La biblioteca no tiene web propia sino un perfil de Facebook, un canal de Youtube y enlaces desde y hacia webs relacio- } \\
\text { nadas como por ejemplo http://www.desdelamina.net. Intenta tener presencia en los medios de comunicación locales y } \\
\text { generales (Soteras, 2013). } \\
\text { Se publican regularmente folletos y otros materiales de difusión adaptados al entorno: bilingües catalán-español y letra } \\
\text { grande. Los carteles se cuelgan por el barrio. } \\
\text { Cada año se publica un libro de forma manual para conmemorar el Día del libro, coincidente con Sant Jordi. En el de } 2014, \\
5^{\circ} \text { año de la biblioteca, los protagonistas fueron los usuarios: éstos escribieron sus impresiones y qué habían representado } \\
\text { para él los cinco años de biblioteca. }\end{array}$ \\
\hline Relaciones públicas & $\begin{array}{l}\text { La biblioteca forma parte de los grupos de trabajo multisectoriales y multidisciplinares del barrio. Al tratarse de una zona } \\
\text { con un alto porcentaje de población gitana, el personal técnico ha realizado cursos de mediación intercultural para poder } \\
\text { asesorar al resto del equipo. }\end{array}$ \\
\hline Marketing directo & $\begin{array}{l}\text { Se realizan buzoneos en el área de influencia con el folleto informativo de la biblioteca. } \\
\text { En Navidad se hacen campañas específicas. En } 2013 \text { fue ... Y un jamón: se entregó un número por cada préstamo de la } \\
\text { biblioteca (por cada acción, no por cada ejemplar) y se sorteó una cesta de Navidad que contenía libros, DVDs, música, } \\
\text { revistas, y un jamón. } \\
\text { En } 2014 \text { el sorteo tuvo la peculiaridad de dar un regalo seguro (que desconocían los usuarios) gracias a la Fundació Forma- } \\
\text { ció i Treball que regaló a la biblioteca } 150 \text { puzzles y juegos educativos; se revisaron y envolvieron como si fueran nuevos. }\end{array}$ \\
\hline \multicolumn{2}{|r|}{ Precio } \\
\hline $\begin{array}{l}\text { Lista de precios } \\
\text { Descuentos } \\
\text { Rebajas } \\
\text { Período de pago } \\
\text { Créditos }\end{array}$ & Coste del servicio por habitante del municipio. \\
\hline
\end{tabular}


- Zona de adultos: atención personalizada, silencio, tertulia literaria, aprendizaje de catalán.

- Zona infantil: atención personalizada.

\subsection{Marketing mix}

En 1962 Jerome McCarthy introdujo el concepto de marketing mix. Se trata del conjunto de medios que utiliza una empresa para alcanzar sus objetivos de marketing en el mercado elegido. Esos medios se pueden clasificar en cuatro grupos básicos que se han llamado las 4P del marketing: producto, precio, lugar (place) y promoción. Según Kotler (2000) cada una de las 4 P tiene asociados otros servicios.

\subsection{Aplicación de marketing mix}

Se pueden mostrar los resultados de la aplicación de las estrategias empresariales con indicadores cuantitativos y cualitativos.

Los primeros son muy útiles para la toma de decisiones, y en especial en entornos económicos, que es donde se inició su aplicación. Sin embargo, no todas las técnicas ofrecen los mismos resultados en función de dónde y cómo se aplican.

Los indicadores cualitativos (tabla 1), que no se pueden mostrar en un gráfico, son el resultado de aplicar diversas estrategias:

- observación y escucha del entorno, de forma objetiva y continua, intentando ser próximos;

- creación de necesidades compartiendo experiencias, mostrando logros, exponiendo resultados, etc.;

- identificación de las personas clave de la comunidad (gitana y no gitana);

- comunicación: atención a las demandas dando respuestas respetando el entorno y las costumbres, sin opinar;

- realización de actividades con las entidades y los habitantes del barrio, no para ellos;

- establecimiento de alianzas y colaboraciones estratégicas, procurando tener presencia física en el barrio y en los medios de comunicación;

- fidelización: cada usuario tiene su espacio, servicio o libro para sus intereses o necesidades. Una muestra del nivel de satisfacción de los usuarios fue la preparación de dulces (panellets) para el personal de la Biblioteca el día de Todos los Santos.

La lección aprendida después de tres años de aplicación de estas estrategias es que no existen métodos replicables en todos los entornos, sino un denominador común que es la necesidad de flexibilidad y adaptación por una parte, y de reinventarse continuamente por otra. De igual importancia es la aplicación del ensayo-error. Una estrategia comercial aplicada el año pasado y con resultados positivos no es garantía de éxito para el año en curso, porque el entorno varía de manera constante, de ahí la necesidad de observar y adaptar el servicio continuamente.

\section{Bibliografía}

Angulo, Silvia; Benvenuty, Luis (2012). "Las obras del complejo universitario del Fòrum de Barcelona arrancan". La vanguardia, 19 de junio. http://www.lavanguardia.com/vida/20120619/54313406178/ obras-complejo-universitario-forum-barcelona.html

El periódico (2012). "La Biblioteca de La Mina sortea un jamón para ganar usuarios". El periódico, 7 de diciembre.

http://www.elperiodico.com/es/noticias/barcelona/ biblioteca-mina-sortea-jamon-para-ganar-usuarios-2267366

Fuller, George (1998). Win-win management. Madrid: Gestión 2000, ISBN: 9788480883337

Kotler, Philip (2000). Dirección de marketing, 10a ed. Madrid: Prentice Hall. ISBN: 9788483222089

La vanguardia (2012). "Sant Adrià acogerá el proyecto Barcelona KEY, la futura ciudad de la economía del conocimiento". La vanguardia, 18 de octubre.

http://www.lavanguardia.com/local/20121018/54352683660/ sant-adria-proyecto-barcelona-key.html

Learned, Edmund P. (1969). Business policy, text and cases. Irwin: Homewood. ISBN: 0256019894

McCarthy, Jerome (1962). Basic marketing: a managerial approach. Homewood, Irwin.

Moya, Mireia (2013). "Es imposible vivir aquí". El periódico, 2 de marzo.

http://www.elperiodico.com/es/noticias/barcelona/ imposible-vivir-aqui-2329741

Placer, David (2010). "La Biblioteca de La Mina trabaja a medio gas por falta de demanda". El periódico, 24 de noviembre. http://www.elperiodico.com/es/noticias/barcelona/20101124/ biblioteca-mina-trabaja-medio-gas-por-falta-demanda/594871. shtml

Santesmases-Mestre, Miguel (2012). Márqueting: conceptos y estrategias. Madrid: Pirámide, pp. 219-243. ISBN: 9788436826135

Soteras, Jordi (2013). "La mina de lectores". El mundo, 2 de diciembre.

http://www.elmundo.es/cataluna/2013/12/08/52a4a28e0 ab74086768b4579.html

\section{Webs}

Canal de YouTube de la Biblioteca Font de La Mina https://www.youtube.com/user/fontdelamina

Degerencia.com

http://www.degerencia.com

Desde La Mina

http://www.desdelamina.net

Facebook de la Biblioteca Font de La Mina https://www.facebook.com/biblioteca.fontdelamina?fref $=$ ts

Keynegotiations (kn)

http://www.keynegotiations.com

OCLC WebJunction

http://www.webjunction.org/marketing

Proven models

http://www.provenmodels.com 